\title{
Physiological and Growth Responses of Six Turfgrass Species Relative to Salinity Tolerance
}

\author{
Md. Kamal Uddin, ${ }^{1}$ Abdul Shukor Juraimi, ${ }^{2}$ Mohd. Razi Ismail, ${ }^{1}$ Md. Alamgir Hossain, ${ }^{1}$ \\ Radziah Othman, ${ }^{3}$ and Anuar Abdul Rahim ${ }^{3}$ \\ ${ }^{1}$ Institute of Tropical Agriculture, Universiti Putra Malaysia, Selangor, 43400 Serdang, Malaysia \\ ${ }^{2}$ Department of Crop Science, Universiti Putra Malaysia, Selangor, 43400 Serdang, Malaysia \\ ${ }^{3}$ Department of Land Management, Universiti Putra Malaysia, 43400 Serdang, Malaysia
}

Correspondence should be addressed to Md. Kamal Uddin, mkuddin07@yahoo.com

Received 13 January 2012; Accepted 26 February 2012

Academic Editor: Pablo Abbate

Copyright (c) $2012 \mathrm{Md}$. Kamal Uddin et al. This is an open access article distributed under the Creative Commons Attribution License, which permits unrestricted use, distribution, and reproduction in any medium, provided the original work is properly cited.

The demand for salinity-tolerant turfgrasses is increasing due to augmented use of effluent or low-quality water (sea water) for turf irrigation and the growing turfgrass industry in coastal areas. Experimental plants, grown in plastic pots filled with a mixture of river sand and $\operatorname{KOSAS}^{\mathrm{R}}$ peat $(9: 1)$, were irrigated with sea water at different dilutions imparting salinity levels of 0,8 , 16, $24,32,40$, or $48 \mathrm{dS} \mathrm{m} \mathrm{m}^{-1}$. Salinity tolerance was evaluated on the basis of leaf firing, shoot and root growth reduction, proline content, and relative water content. Paspalum vaginatum was found to be most salt tolerant followed by Zoysia japonica and Zoysia matrella, while Digitaria didactyla, Cynodon dactylon "Tifdwarf," and Cynodon dactylon "Satiri" were moderately tolerant. The results indicate the importance of turfgrass varietal selection for saline environments.

\section{Introduction}

Salinity is a major abiotic environmental stress that is reported to be responsible for reducing plant growth across the globe. Sea water intrusion, in coastal states, has imposed salinity problems in turfgrass culture $[1,2]$. Sodium chloride $(\mathrm{NaCl})$ is the major compound contributing salinity in soils, and more salt-tolerant turfgrasses are required to cope this problem [3]. Therefore, development of salt-tolerant turfgrasses is becoming increasingly necessary in many parts of the world including Malaysia. Salt accumulation in soils, limitations on use of groundwater, and salt water intrusion into groundwater may restrict cultivation of glycophytic crops in these areas [4]. Salinity lowers water potential and restricts of water to plants [5]. Presence of excessive salt $(\mathrm{NaCl})$ outside the cell can induce an osmotic stress, which may adversely affect the plant growth [6]. Hence, osmotic balance or osmoregulation is certainly a crucial factor for the survival of a plant under salt-stressed conditions. Generally, plants have developed different adaptive mechanisms to mitigate salinity under the saline environments [7-9]. Among these, salt exclusion is considered to be the most important adaptive feature of nonhalophytic plants, whilst most tolerant halophytes are salt accumulators [5]. Salt-accumulating halophytes are very crucial for osmotic adjustment. It could be achieved in the following ways: (i) by accumulating inorganic osmolyte $\left(\mathrm{K}^{+}\right)$and/or (ii) accumulating organic osmolytes such as proline. Therefore, salt-tolerant halophytic plants have the capability to minimize the detrimental effects by morphological means and physiological or biochemical processes [10].

Some of the turfgrass species are halophytic in nature. So salt-tolerant turf varieties would allow landscape development in saline environments and would be ideal in such environments, where limited or no fresh water is available for irrigation and salt water is the only option for irrigation practices. In addition, the use of sea water is also a good strategy for weed control in seashore paspalum worldwide. 
TABLE 1: Turfgrass species used in this study.

\begin{tabular}{lll}
\hline Scientific name & Common name & Salt tolerance \\
\hline Paspalum vaginatum Sw. & Seashore paspalum & Salt tolerant \\
Zoysia japonica Steud. & Japanese lawn grass & Salt tolerant \\
Zoysia matrella (L.) Merrill & Manila grass & Salt tolerant \\
Cynodon dactylon $x$. Cynodon transvaalensis. & Hybridbermuda grass (Satiri) & Medium salt tolerant \\
Cynodon dactylon x. Cynodon transvaalensis. & Hybridbermuda grass (Tifdwarf) & Medium salt tolerant \\
Digitaria didactyla Willd. & Serangoon grass & Medium salt tolerant \\
\hline
\end{tabular}

The native bermudagrass (Cynodon dactylon) here is quite salt-tolerant and grows vigorously, other salt-tolerant turfgrass species may also grow in the saline environments. In our previous reports $[11,12]$, several turfgrass species were identified in the coastal areas of Malaysia. Interestingly, the development of turfgrass industry especially in the coastal areas of Malaysia is an emerging field. To the best of our knowledge, published literatures are very scanty on salt tolerance studies in turfgrass species, which have been or being conducted in Malaysia. Therefore, this study was framed to determine the relative salinity tolerance and growth response of six important turfgrass species to salinity.

\section{Materials and Methods}

Glasshouse experiments were conducted at Faculty of Agriculture, University Putra Malaysia. Plastic pots $(14 \times 15 \mathrm{~cm})$ were filled up with sandy soil (a mixture of river sand and peat; $9: 1, \mathrm{v} / \mathrm{v}$ ). The sandy soil had electrical conductivity (EC) $0.3 \mathrm{dS} \mathrm{m}^{-1}$, organic carbon $0.69 \%$, sand $97.93 \%$, silt $1.89 \%$, and clay $0 \%$ with $\mathrm{pH} 5.23$. The glasshouse temperature, relative humidity, and light intensity in morning time were $32^{\circ} \mathrm{C}, 80 \%$, and 110 micromol m $\mathrm{m}^{-2} \mathrm{~s}^{-1}$, and after noon $36^{\circ} \mathrm{C}, 70 \%$, and 175 micromol m $\mathrm{m}^{-2} \mathrm{~s}^{-1}$, respectively. The temperature was measured using a laboratory thermometer, and light intensity was monitored using a heavy duty light meter (Extech model 407026). Based on earlier findings of $[13,14]$, the three most salt-tolerant and three medium salt tolerant turfgrass species (Table 1) were used in this study.

The native soil was washed off the sods, and the sods were then transplanted into the plastic pots and grown for 8 weeks under nonsaline irrigation to achieve full growth. Three plants were transplanted in each pot. All species were narrow leaf and were clipped weekly at a cutting height of $5 \mathrm{~mm}$. After 8 weeks thereafter, salinity treatments were initiated. Salinity treatments of $0,8,16,24,32,40$, and $48 \mathrm{dS} \mathrm{m}^{-1}$ (sea water) were applied. The control grasses were irrigated with distilled water. Sea water was diluted by adding distilled water to achieve different treatments. To avoid salinity shock, salinity levels were increased gradually by $8 \mathrm{dSm}^{-1}$ day $^{-1}$ for each treatment until the final salinity levels were achieved. After that, irrigation water was applied daily upto four weeks. The amount of water applied was $200 \mathrm{~mL}$ per pot. Data on leaf firing, proline, chlorophyll, relative water content, shoot and root dry weight were recorded 4 weeks after application of salinity treatment.
2.1. Determination of Leaf Firing. Leaf firing was estimated as total percentage of chlorotic leaf area, with $0 \%$ corresponding to no leaf firing and $100 \%$ for total brown leaves [15].

2.2. Determination of Shoot and Root Dry Weight. At the end of experiment (four weeks after salt initiation), shoots above the soil surface were harvested and washed with tap water and then distilled water to remove all soil particles. After harvesting the shoots, roots were removed from the soil, washed with tap water, and rinsed with distilled water. The shoot and root samples were then oven-dried to a constant weight at $70^{\circ} \mathrm{C}$ for 3 days. The dry weight (g/plant) was recorded for each treatment.

2.3. Determination of Proline Content. Proline was estimated following method of [16]. Fresh leaf tissue $(0.5 \mathrm{~g})$ was homogenized in $10 \mathrm{~mL}$ of $3 \%$ sulfosalicylic acid, and the homogenate was filtered through Whatman no. 2 filter paper. Two milliliters of the filtrate were brought to reaction with $2 \mathrm{~mL}$ acid ninhydrin solution $(1.25 \mathrm{~g}$ ninhydrin in $30 \mathrm{~mL}$ glacial acetic acid), $20 \mathrm{~mL}$ orthophosphoric acid $(6 \mathrm{M})$, and $2 \mathrm{~mL}$ of glacial acetic acid for $1 \mathrm{~h}$ at $100^{\circ} \mathrm{C}$. The reaction was terminated in an ice bath. The reaction mixture was extracted with $4 \mathrm{~mL}$ toluene, mixed vigorously by passing a continuous stream of air for 1-2 min. The chromophore containing toluene was aspirated from the aqueous phase, warmed at room temperature, and the absorbance was recorded spectrophotometrically (Model UV-3101PC, UV-VIS NIR) at $520 \mathrm{~nm}$. The proline concentration was determined from a standard curve and calculated on fresh weight basis as follows:

$\mu$ mol proline $\mathrm{g}^{-1}$ fresh weight

$$
=\frac{\mu \mathrm{g} \text { proline } \mathrm{mL}^{-1} \times \mathrm{mL} \text { of toluene/115.5 }}{\mathrm{g} \text { of sample }} .
$$

2.4. Determination of Chlorophyll Content. Chlorophyll content was estimated following method of [17]. Fresh leaves, from each pot, were cut into small pieces using a scissors and $200 \mathrm{mg}$ of cut leaves were transferred into a plastic vial containing $20 \mathrm{~mL}$ of $80 \%$ acetone. The vial was quickly corked airtight and kept in the dark for $72 \mathrm{~h}$. Absorbance of the solution was recorded at 645 and $663 \mathrm{~nm}$ spectrophotometrically (Model UV-3101PC, UV-VIS NIR). Chlorophyll 
TABLE 2: Main effect and interaction effect on different variables by salinity and species.

\begin{tabular}{lccc}
\hline Variable & Salinity & Species & Salinity $\times$ species \\
\hline Leaf firing & $1665.78^{* * *}$ & $513.16^{* * *}$ & $75.83^{* * *}$ \\
Shoot dry weight & $95.82^{* * *}$ & $1317.65^{* * *}$ & $4.01^{* * *}$ \\
Root dry weight & $79.83^{* * *}$ & $287.54^{* * *}$ & $1.15 \mathrm{~ns}$ \\
Proline & $2176.10^{* * *}$ & $585.87^{* * *}$ & $58.07^{* * *}$ \\
Relative water content & $78.07^{* * *}$ & $13.85^{* * *}$ & $1.45 \mathrm{~ns}$ \\
Chlorophyll-a & $30.03^{* * *}$ & $152.19^{* * *}$ & $0.89 \mathrm{~ns}$ \\
Chlorophyll-b & $67.91^{* * *}$ & $78.03^{* * *}$ & $4.20^{* * *}$ \\
Total chlorophyll & $65.86^{* * *}$ & $206.75^{* * *}$ & $2.13^{* * *}$ \\
\hline
\end{tabular}

Numbers are $F$ values significant at $* * * P<0.0001$, ns: not significant.

content was estimated and expressed as $\mathrm{mg} \mathrm{g}^{-1}$ of sample using the following formulae:

Chlorophyll a content $(\mathrm{mg} / \mathrm{g}$ fresh leaf)

$$
=\frac{12.7\left(A_{663}\right)-2.69\left(A_{645}\right)}{1000} \times \frac{V}{W}
$$

Chlorophyll b content $(\mathrm{mg} / \mathrm{g}$ fresh leaf)

$$
=\frac{22.9\left(A_{645}\right)-4.86\left(A_{663}\right)}{1000} \times \frac{V}{W}
$$

Total chlorophyll content (mg/g fresh leaf)

$$
=\frac{20.2\left(A_{645}\right)+8.02\left(A_{663}\right)}{1000} \times \frac{V}{W}
$$

where $A_{645}$ and $A_{663}$ represent absorbance of solution at 645 and $663 \mathrm{~nm}$, respectively, $V$ : volume of the solution in $\mathrm{mL}$, $W$ : weight of fresh leaf sample in gram, 12.7, 2.69, 22.9, 4.86, 20.2 , and 8.02 are absorption coefficients.

2.5. Determination of Relative Water Content. Relative water content (RWC) was determined as described by [18] on leaf tissues excised in the morning (around $9.00 \mathrm{am}$ ). Excised leaves from each pot $(0.2 \mathrm{~g})$ were measured for fresh weight $(\mathrm{FW})$, and leaf samples were rehydrated in a water-filled petri dish for $4 \mathrm{~h}$ at room temperature. Turgor weight (TW) was measured by allowing full rehydration, removing all water from leaf surface, and weighing. Leaf dry weights were recorded after oven drying for one week at $60^{\circ} \mathrm{C}$. The leaf relative water content was determined using the following formula:

$$
\text { RWC }=\frac{\text { Fresh weight }- \text { Dry weight }}{\text { Fully turgid weight }- \text { Dry weight }} \times 100 .
$$

2.6. Root Histology Using Scanning Electron Microscopy. Roots were sampled from two root zones (root tips at 0-50 $\mathrm{mm}$ from tip, and mature roots) and were cut into $5 \mathrm{~mm}$ portions with a sharp blade. The excised roots were placed in formalin acetic acid (FAA) and vacuumed for $1 \mathrm{~h}$ at $650 \mathrm{~mm} \mathrm{Hg}$. Specimens were postfixed in $1 \%$ osmium tetraoxide for $2 \mathrm{~h}$, dehydrated for $30 \mathrm{~min}$ in each graded ethanol series at 30, 50, 70,90, 95, and 100\%, and dried in
Baltec CPD 030 critical point dryer apparatus. The tissues were mounted on stubs, coated with gold using auto fine coater (JEOL JFC-1600, Japan) for $20 \mathrm{~min}$, and viewed under a scanning electron microscope (JEOL JSM-5610LV, Japan), at high vacuum and acceleration voltage of $15 \mathrm{kV}$ with a working distance of $23 \mathrm{~mm}$.

2.7. Statistical Analysis. Data were analyzed statistically following randomized complete block design using ANOVA procedure in SAS statistical software (SAS). The treatment means were compared using protected least significant differences (LSD) at 5\% level. Data of leaf firing was proportionate, so arcsine square root transformation was done.

\section{Results}

3.1. Leaf Firing. Interaction of salinity and species had a significant effect on leaf firing (Table 2). Leaf firing (\%) increased with increasing salinity in all turfgrass species (Table 3). However, comparatively less salinity injury was recorded in $P$. vaginatum, $Z$. japonica, and $Z$. matrella compared to D. didactyla, $C$. dactylon "Tifdwarf" and $C$. dactylon "Satiri" at all salinity levels. There was no injury (0\%) recorded in all species up to $16 \mathrm{dS} \mathrm{m}^{-1}$ salinity, except for $D$. didactyla and $C$. dactylon "Tifdwarf" which showed light injury symptoms of 5 and $8 \%$, respectively. At $24 \mathrm{dS} \mathrm{m}^{-1}$, the highest injury (25\%) was recorded in $D$. didactyla, while the lowest injury of $5 \%$ was observed in $P$. vaginatum. At $32 \mathrm{dS} \mathrm{m}^{-1}$, leaf firing drastically increased to 79 and $75 \%$ in D. didactyla and C. dactylon "Tifdwarf," respectively. At the highest salinity level of $48 \mathrm{dS} \mathrm{m}^{-1}$, the least leaf firing was observed in P. vaginatum (15\%) followed by $Z$. japonica $(25 \%)$ and $Z$. matrella $(39 \%)$ compared to 80-100\% leaf firing in D. didactyla, C. dactylon "Tifdwarf," and C. dactylon "Satiri." Overall, the highest leaf firing was recorded in D. didactyla, while the lowest in P. vaginatum.

3.2. Shoot Dry Weight. Interaction effect of salinity and species was significant $(P<0.05)$ on shoot dry weight (Table 2). Shoot dry weights (SDWs) of turfgrass species decreased as the level of salinity increased (Figure 1). Results showed that $P$. vaginatum was the most salt-tolerant species 
TABLE 3: Effect of salinity on leaf firing of six turfgrass species.

\begin{tabular}{|c|c|c|c|c|c|c|c|}
\hline \multirow{2}{*}{$\begin{array}{l}\mathrm{EC}_{w} \\
\left(\mathrm{dS} \mathrm{m}^{-1}\right)\end{array}$} & \multicolumn{7}{|c|}{ Turfgrass species (\% leaf firing) } \\
\hline & P. vaginatum & Z. japonica & Z. matrella & D. didactyla & $\begin{array}{l}\text { C. dactylon } \\
\text { "Tifdwarf" }\end{array}$ & $\begin{array}{l}\text { C. dactylon } \\
\text { "Satiri" }\end{array}$ & $\operatorname{LSD}(0.05)$ \\
\hline 0 & 0 e $(0.28)$ & 0 e $(0.28)$ & 0 e $(0.28)$ & $0 \mathrm{f}(0.28)$ & $0 \mathrm{f}(0.28)$ & 0 e $(0.28)$ & 0.00 \\
\hline 8 & 0 e $(0.28)$ & 0 e $(0.28)$ & 0 e $(0.28)$ & $0 \mathrm{f}(0.28)$ & $0 \mathrm{f}(0.28)$ & 0 e $(0.28)$ & 0.00 \\
\hline 16 & 0 e $(0.28)$ & 0 e $(0.28)$ & 0 e $(0.28)$ & 5 e (12.79) & 8 e (16.37) & $0 \mathrm{e}(0.28)$ & 2.45 \\
\hline 24 & $5 \mathrm{~d}(12.89)$ & $10 \mathrm{~d}(18.26)$ & $15 \mathrm{~d}(22.65)$ & $25 \mathrm{~d}(29.90)$ & $18 \mathrm{~d}(25.01)$ & $15 \mathrm{~d}(22.65)$ & 4.31 \\
\hline 32 & $8 \mathrm{c}(16.37)$ & $15 c(22.65)$ & $20 c(26.49)$ & $79 \mathrm{c}(63.17)$ & 45 c (42.14) & 25 c (29.95) & 2.57 \\
\hline 40 & 12 b (20.20) & $20 \mathrm{~b}(26.52)$ & $26 \mathrm{~b}(30.64)$ & $93 \mathrm{~b}(76.80)$ & $85 \mathrm{~b}(67.39)$ & $69 \mathrm{~b}(56.37)$ & 4.32 \\
\hline 48 & $15 \mathrm{a}(22.65)$ & $25 \mathrm{a}(29.95)$ & $39 \mathrm{a}(38.64)$ & $100 \mathrm{a}(89.75)$ & $94 \mathrm{a}(77.81)$ & $80 \mathrm{a}(63.83)$ & 4.52 \\
\hline LSD (0.05) & 2.31 & 2.34 & 2.30 & 6.19 & 4.36 & 5.11 & \\
\hline
\end{tabular}

Means within columns followed by the same letter are not significantly different at $P=0.05$ (LSD test).

Values in the parentheses indicate transformed by Arcsine square root.

TABLE 4: Effect of salinity on leaf proline content of six turfgrass species.

\begin{tabular}{|c|c|c|c|c|c|c|c|}
\hline \multirow{2}{*}{$\begin{array}{l}\mathrm{EC}_{w} \\
\left(\mathrm{dS} \mathrm{m}^{-1}\right)\end{array}$} & \multicolumn{7}{|c|}{ Turfgrass species (proline contents in $\mathrm{mg} \mathrm{g}^{-1}$, fresh weight) } \\
\hline & P. vaginatum & Z. japonica & Z. matrella & D. didactyla & $\begin{array}{l}\text { C. dactylon } \\
\text { "Tifdwarf" }\end{array}$ & $\begin{array}{l}\text { C. dactylon } \\
\text { "Satiri" }\end{array}$ & $\operatorname{LSD}(0.05)$ \\
\hline 0 & $3.33 \mathrm{f}$ & $3.60 \mathrm{~d}$ & $3.67 \mathrm{f}$ & $3.55 \mathrm{e}$ & $5.60 \mathrm{f}$ & $6.35 \mathrm{e}$ & 0.96 \\
\hline 8 & 4.60 ef (1.4) & $4.07 \mathrm{~d}(1.1)$ & $4.62 \mathrm{f}(1.3)$ & 6.42 e (1.8) & $7.25 \mathrm{f}(1.3)$ & 10.60 e (1.7) & 2.31 \\
\hline 16 & 7.80 ed (2.3) & $6.50 \mathrm{~d}(1.8)$ & $6.02 \mathrm{e}(1.7)$ & $12.40 \mathrm{~d}(3.5)$ & 15.35 e (2.7) & $29.90 \mathrm{~d}(4.7)$ & 3.65 \\
\hline 24 & $11.61 \mathrm{~d}(3.5)$ & 13.10 c (3.6) & $9.24 \mathrm{~d}(2.5)$ & $15.05 \mathrm{~d}(4.2)$ & $26.35 \mathrm{~d}(4.7)$ & $52.50 \mathrm{c}(8.3)$ & 1.77 \\
\hline 32 & 26.90 c (8.1) & 16.25 c (4.5) & 11.30 c (3.1) & 34.55 c (9.7) & 37.57 c (6.7) & $66.52 \mathrm{~b}(10.5)$ & 3.53 \\
\hline 40 & $51.20 \mathrm{~b}(15.4)$ & $45.82 \mathrm{~b}(12.7)$ & $25.57 \mathrm{~b}(7.0)$ & $43.27 \mathrm{~b}(12.2)$ & $65.15 \mathrm{~b}(11.2)$ & 71.35 a $(11.2)$ & 4.09 \\
\hline 48 & 77.90 a $(23.4)$ & $49.62 \mathrm{a}(13.8)$ & $43.52 \mathrm{a}(12.0)$ & 49.92 a $(14.1)$ & 62.57 a $(11.6)$ & $74.85 \mathrm{a}(11.8)$ & 5.18 \\
\hline $\operatorname{LSD}(0.05)$ & 4.45 & 3.26 & 1.26 & 3.49 & 1.93 & 4.43 & \\
\hline
\end{tabular}

Means within columns followed by the same letter are not significantly different at $P=0.05$ (LSD test).

Values in the parentheses indicate $\mathrm{x}$-fold increase relative to the control.

being statistically significant with others. At the highest salinity level $\left(48 \mathrm{dS} \mathrm{m}^{-1}\right)$, SDW reduction in P. vaginatum was only $23 \%$ relative to control treatment. Zoysia japonica followed a similar trend as $P$. vaginatum for salinities upto $24 \mathrm{dS} \mathrm{m}^{-1}$. At $48 \mathrm{dS} \mathrm{m}^{-1}$, significantly higher SDW reductions were observed in D. didactyla (51\%), C. dactylon "Tifdwarf" (53\%), and C. dactylon "Satiri" (44\%).

3.3. Root Dry Weight. The results showed that root dry weight (RDW) significantly $(P<0.05)$ decreased with increasing salinity (Figure 2). At $16 \mathrm{dS} \mathrm{m}^{-1}$, a significant difference was noted among the species. However, $P$. vaginatum, C. dactylon "Tifdwarf," Z. japonica, and Z. matrella produced greater RDW than the others at $24 \mathrm{dS} \mathrm{m}^{-1}$ salinity. At the highest salinity $\left(48 \mathrm{dS} \mathrm{m}^{-1}\right)$, RDW reduction was least in $P$. vaginatum (34\%) followed by $Z$. japonica (46\%); while highest in C. dactylon "Tifdwarf" (67\%) followed by $C$. dactylon "Satiri" (54\%), Z. matrella (53\%), and D. didactyla $(47 \%)$. However, there were nonsignificant effect on root dry matter yield when salinity and species were interacted (Table 2).
3.4. Leaf Proline Content. Proline accumulation in the leaves of all turfgrass species increased with increasing salinity (Table 4). There were two distinct trends in proline accumulation among the species analyzed. In all turfgrass species (except C. dactylon "Satiri"), proline accumulation increased gradually up to $24 \mathrm{dS} \mathrm{m}^{-1}$ but increased abruptly at 32 and $48 \mathrm{dS} \mathrm{m}^{-1}$. At $48 \mathrm{dS} \mathrm{m}^{-1}$, a significantly higher (23.4-folds over the control) accumulation of proline was observed in P. vaginatum compared to in C. dactylon "Tifdwarf" (11.6-folds). There was a difference between the grasses with respect to proline accumulation patterns at 32 and $48 \mathrm{dS} \mathrm{m}^{-1}$. On the basis of proline accumulation ability, turfgrass species were ranked as $P$. vaginatum $>Z$. matrella $>$ $D$. didactyla $>Z$. japonica $>$ both of the $C$. dactylon entries. Interaction between salinity and species had also a significant $(P<0.001)$ effect on proline level (Table 7$)$.

3.5. Leaf Relative Water Content (RWC). Interaction effect of salinity and species was not significant for relative water content (Table 5). Relative water content (RWC) of all turfgrass species was significantly $(P<0.05)$ influenced by salinity. As salinity increased, RWC decreased. However, 
TABLE 5: Effect of salinity on leaf relative water content of six turfgrass species.

\begin{tabular}{|c|c|c|c|c|c|c|c|}
\hline \multirow{2}{*}{$\begin{array}{l}\mathrm{EC}_{w} \\
\left(\mathrm{dS} \mathrm{m}^{-1}\right)\end{array}$} & \multicolumn{7}{|c|}{ Turfgrass species (relative water contents in $\%$, fresh weight) } \\
\hline & P. vaginatum & Z. japonica & Z. matrella & D. didactyla & $\begin{array}{l}\text { C. dactylon } \\
\text { "Tifdwarf" }\end{array}$ & $\begin{array}{l}\text { C. dactylon } \\
\text { "Satiri" }\end{array}$ & $\operatorname{LSD}(0.05)$ \\
\hline 0 & $93.16 \mathrm{a}$ & $89.48 \mathrm{a}$ & 89.89 a & $87.33 \mathrm{a}$ & $90.85 \mathrm{a}$ & 90.18 a & 8.83 \\
\hline 8 & $90.24 \mathrm{ab}$ & $87.57 \mathrm{a}$ & 90.97 a & $86.28 \mathrm{a}$ & $90.14 \mathrm{a}$ & 90.39 a & 6.29 \\
\hline 16 & $90.23 \mathrm{ba}$ & $85.22 \mathrm{a}$ & 86.87 a & $84.78 \mathrm{a}$ & $85.02 \mathrm{ba}$ & 86.19 a & 6.64 \\
\hline 24 & 87.84 ba & $84.92 \mathrm{a}$ & $88.51 \mathrm{a}$ & $82.42 \mathrm{a}$ & 83.91 ba & $78.59 \mathrm{~b}$ & 9.92 \\
\hline 32 & $86.04 \mathrm{~b} \mathrm{bc}$ & $78.39 \mathrm{~b}$ & $84.09 \mathrm{a}$ & $68.06 \mathrm{~b}$ & $78.70 \mathrm{~b}$ & $76.42 \mathrm{~b}$ & 9.97 \\
\hline 40 & $79.77 \mathrm{dc}$ & $72.28 \mathrm{c}$ & $73.28 \mathrm{~b}$ & $63.46 \mathrm{~b}$ & $65.27 \mathrm{c}$ & $64.85 \mathrm{~b}$ & 9.51 \\
\hline 48 & $78.68 \mathrm{~b}$ & $66.30 \mathrm{~d}$ & $64.98 \mathrm{c}$ & $55.35 \mathrm{c}$ & $62.51 \mathrm{c}$ & $57.30 \mathrm{c}$ & 8.03 \\
\hline LSD $(0.05)$ & 6.49 & 5.94 & 8.05 & 5.85 & 8.76 & 6.97 & \\
\hline
\end{tabular}

Means within columns followed by the same letter are not significantly different at $P=0.05$ (LSD test).

TABLE 6: Effect of salinity on chlorophyll-a concentration of six turfgrass species.

\begin{tabular}{|c|c|c|c|c|c|c|c|}
\hline \multirow{2}{*}{$\begin{array}{l}\mathrm{EC}_{w} \\
\left(\mathrm{dS} \mathrm{m}^{-1}\right)\end{array}$} & \multicolumn{7}{|c|}{ Turfgrass species (chlorophyll-a contents in $\mathrm{mg} \mathrm{g}^{-1}$, fresh weight) } \\
\hline & P. vaginatum & Z. japonica & Z. matrella & D. didactyla & $\begin{array}{l}\text { C. dactylon } \\
\text { "Tifdwarf" }\end{array}$ & $\begin{array}{l}\text { C. dactylon } \\
\text { "Satiri" }\end{array}$ & $\operatorname{LSD}(0.05)$ \\
\hline 0 & $0.49 \mathrm{a}$ & $0.40 \mathrm{a}$ & $0.36 \mathrm{a}$ & $0.30 \mathrm{a}$ & $0.49 \mathrm{a}$ & $0.57 \mathrm{a}$ & 0.070 \\
\hline 8 & $0.47 \mathrm{a}$ & $0.39 \mathrm{ab}$ & $0.33 \mathrm{ab}$ & $0.29 \mathrm{a}$ & $0.48 \mathrm{a}$ & $0.57 \mathrm{a}$ & 0.059 \\
\hline 16 & $0.46 \mathrm{ab}$ & $0.39 \mathrm{ab}$ & $0.31 \mathrm{abc}$ & $0.27 \mathrm{a}$ & $0.46 \mathrm{a}$ & $0.56 \mathrm{a}$ & 0.067 \\
\hline 24 & $0.45 \mathrm{abc}$ & $0.38 \mathrm{ab}$ & $0.30 \mathrm{abc}$ & $0.26 \mathrm{a}$ & $0.45 \mathrm{ab}$ & $0.55 \mathrm{a}$ & 0.080 \\
\hline 32 & $0.42 \mathrm{bc}$ & $0.35 \mathrm{bc}$ & $0.29 \mathrm{bc}$ & $0.20 \mathrm{~b}$ & $0.40 \mathrm{bc}$ & $0.53 \mathrm{a}$ & 0.079 \\
\hline 40 & $0.41 \mathrm{c}$ & $0.33 \mathrm{~cd}$ & $0.26 \mathrm{c}$ & $0.19 \mathrm{~b}$ & $0.35 \mathrm{~cd}$ & $0.45 \mathrm{~b}$ & 0.061 \\
\hline 48 & $0.40 \mathrm{c}$ & $0.30 \mathrm{~d}$ & $0.24 \mathrm{c}$ & $0.12 \mathrm{c}$ & $0.31 \mathrm{~d}$ & $0.41 \mathrm{~b}$ & 0.065 \\
\hline $\operatorname{LSD}(0.05)$ & 0.051 & 0.042 & 0.066 & 0.063 & 0.052 & 0.077 & \\
\hline
\end{tabular}

Means within columns followed by the same letter are not significantly different at $P=0.05$ (LSD test).

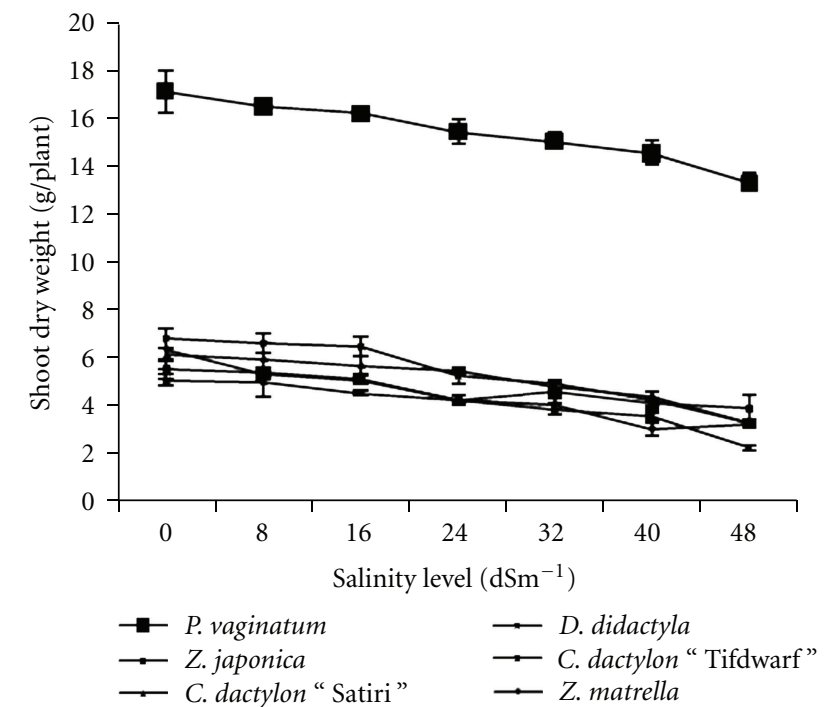

FIGURE 1: Shoot dry weight at different salinity levels of six turfgrass species.

RWC for most of the species did not change up to $24 \mathrm{dS} \mathrm{m}^{-1}$ compared to the control (Table 5). Relative water content significantly decreased at $32 \mathrm{dS} \mathrm{m}^{-1}$ salinity level, except for C. dactylon "Satiri" and Z. matrella. According to reduction

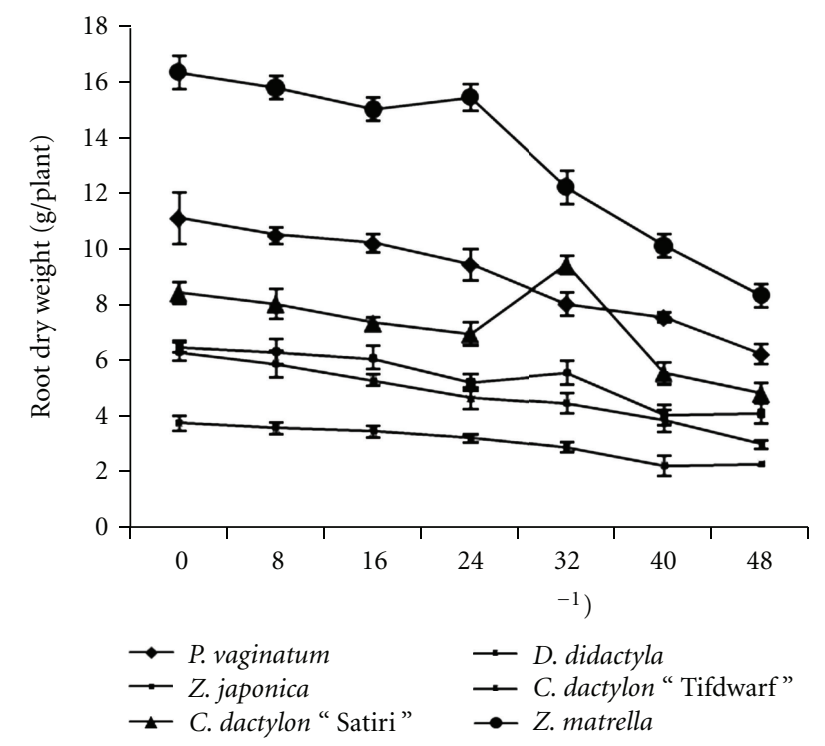

FIGURE 2: Root dry weight at different salinity levels of six turfgrass species.

in RWC at $48 \mathrm{dS} \mathrm{m}^{-1}$ salinity level, species were ranked as D. didactyla $(44.6 \%)>$ C. dactylon "Satiri" $(42.7 \%)>C$. 
TABLE 7: Effect of salinity on chlorophyll-b concentration of six turfgrass species.

\begin{tabular}{|c|c|c|c|c|c|c|c|}
\hline \multirow{2}{*}{$\begin{array}{l}\mathrm{EC}_{w} \\
\left(\mathrm{dS} \mathrm{m}^{-1}\right)\end{array}$} & \multicolumn{7}{|c|}{ Turfgrass species (chlorophyll-b contents in $\mathrm{mg}^{-1}$, fresh weight) } \\
\hline & P. vaginatum & Z. japonica & Z. matrella & D. didactyla & $\begin{array}{l}\text { C. dactylon } \\
\text { "Tifdwarf" }\end{array}$ & $\begin{array}{l}\text { C. dactylon } \\
\text { "Satiri" }\end{array}$ & $\operatorname{LSD}(0.05)$ \\
\hline 0 & $0.14 \mathrm{a}$ & $0.13 \mathrm{a}$ & $0.12 \mathrm{a}$ & $0.15 \mathrm{a}$ & $0.20 \mathrm{a}$ & $0.20 \mathrm{a}$ & 0.031 \\
\hline 8 & $0.13 \mathrm{ab}$ & $0.13 \mathrm{a}$ & $0.11 \mathrm{~b}$ & $0.13 \mathrm{ab}$ & $0.19 \mathrm{ab}$ & $0.19 \mathrm{ab}$ & 0.022 \\
\hline 16 & $0.12 \mathrm{ab}$ & $0.10 \mathrm{~b}$ & $0.10 \mathrm{~b}$ & $0.13 \mathrm{ab}$ & $0.18 \mathrm{~b}$ & $0.19 \mathrm{ab}$ & 0.017 \\
\hline 24 & $0.12 \mathrm{ab}$ & $0.10 \mathrm{~b}$ & $0.10 \mathrm{bc}$ & $0.12 \mathrm{ab}$ & $0.18 \mathrm{~b}$ & $0.18 \mathrm{~b}$ & 0.019 \\
\hline 32 & $0.11 \mathrm{~b}$ & $0.08 \mathrm{~b}$ & $0.09 \mathrm{~cd}$ & $0.10 \mathrm{bc}$ & $0.12 \mathrm{c}$ & $0.12 \mathrm{c}$ & 0.023 \\
\hline 40 & $0.11 \mathrm{~b}$ & $0.08 \mathrm{~b}$ & $0.08 \mathrm{de}$ & $0.09 \mathrm{bc}$ & $0.09 \mathrm{~d}$ & $0.11 \mathrm{~cd}$ & 0.020 \\
\hline 48 & $0.12 \mathrm{ab}$ & $0.08 \mathrm{~b}$ & $0.06 \mathrm{e}$ & $0.08 \mathrm{c}$ & $0.11 \mathrm{~cd}$ & $0.09 \mathrm{c}$ & 0.019 \\
\hline LSD (0.05) & 0.024 & 0.026 & 0.014 & 0.033 & 0.018 & 0.018 & \\
\hline
\end{tabular}

Means within columns followed by the same letter are not significantly different at $P=0.05$ (LSD test).

TABLE 8: Effect of salinity on total chlorophyll concentration of six turfgrass species.

\begin{tabular}{|c|c|c|c|c|c|c|c|}
\hline \multirow{2}{*}{$\begin{array}{l}\mathrm{EC}_{w} \\
\left(\mathrm{dS} \mathrm{m}^{-1}\right)\end{array}$} & \multicolumn{7}{|c|}{ Turfgrass species (total chlorophyll contents in $\mathrm{mg} \mathrm{g}^{-1}$, fresh weight) } \\
\hline & P. vaginatum & Z. japonica & Z. matrella & D. didactyla & $\begin{array}{l}\text { C. dactylon } \\
\text { "Tifdwarf" }\end{array}$ & $\begin{array}{l}\text { C. dactylon } \\
\text { "Satiri" }\end{array}$ & $\operatorname{LSD}(0.05)$ \\
\hline 0 & $0.62 \mathrm{a}$ & $0.53 \mathrm{a}$ & $0.48 \mathrm{a}$ & $0.45 \mathrm{a}$ & $0.68 \mathrm{a}$ & $0.77 \mathrm{a}$ & 0.069 \\
\hline 8 & $0.60 \mathrm{a}$ & $0.52 \mathrm{a}$ & $0.44 \mathrm{ab}$ & $0.42 \mathrm{ab}$ & $0.67 \mathrm{a}$ & $0.76 \mathrm{a}$ & 0.065 \\
\hline 16 & $0.59 \mathrm{ab}$ & $0.49 \mathrm{a}$ & $0.41 \mathrm{~b}$ & $0.40 \mathrm{ab}$ & $0.66 \mathrm{a}$ & $0.74 \mathrm{a}$ & 0.068 \\
\hline 24 & $0.57 \mathrm{bac}$ & $0.48 \mathrm{ab}$ & $0.40 \mathrm{bc}$ & $0.38 \mathrm{~b}$ & $0.63 \mathrm{ab}$ & $0.73 \mathrm{a}$ & 0.075 \\
\hline 32 & $0.53 \mathrm{bc}$ & $0.41 \mathrm{bc}$ & $0.37 \mathrm{bc}$ & $0.29 \mathrm{c}$ & $0.52 \mathrm{bc}$ & $0.65 \mathrm{~b}$ & 0.083 \\
\hline 40 & $0.52 \mathrm{c}$ & $0.43 \mathrm{dc}$ & $0.34 \mathrm{~cd}$ & $0.29 \mathrm{c}$ & $0.45 \mathrm{c}$ & $0.54 \mathrm{c}$ & 0.064 \\
\hline 48 & $0.52 \mathrm{c}$ & $0.38 \mathrm{~d}$ & $0.31 \mathrm{~d}$ & $0.20 \mathrm{~d}$ & $0.42 \mathrm{c}$ & $0.52 \mathrm{c}$ & 0.068 \\
\hline LSD (0.05) & 0.060 & 0.050 & 0.079 & 0.068 & 0.100 & 0.074 & \\
\hline
\end{tabular}

Means within columns followed by the same letter are not significantly different at $P=0.05$ (LSD test).

dactylon "Tifdwarf” $(37.5 \%)>Z$. matrella $(35.0 \%)>Z$. japonica $(33.7 \%)>$ P. vaginatum $(21.3 \%)$.

3.6. Leaf Chlorophyll Content. Interaction effect of salinity and species was not significant for chlorophyll-a content (Table 7). Increasing salinity up to $24 \mathrm{dS} \mathrm{m}^{-1}$ did not affect chlorophyll-a content (Table 6). There were also no differences between 40 and $48 \mathrm{dS} \mathrm{m}^{-1}$ treatments on chlorophyllb content, except for $D$. didactyla. In $P$. vaginatum, the chlorophyll-b content $\left(0.11 \mathrm{mg} \mathrm{g}^{-1} \mathrm{FW}\right)$ at 32 and $40 \mathrm{dS} \mathrm{m}^{-1}$ salinity levels was significantly different from other salinity levels (average $0.126 \mathrm{mg} \mathrm{g}^{-1} \mathrm{FW}$ ) (Table 7). In Z. japonica, a significant reduction in chlorophyll-b content was observed at $16 \mathrm{dS} \mathrm{m}^{-1}$, but there were no further reductions with increasing salinity.

Total chlorophyll content decreased under salt stress in different turfgrass species (Table 8). Interaction effect of salinity and species was significant $(P<0.05)$ for total chlorophyll (Table 7). Turf species with higher chlorophylla and chlorophyll-b contents, under control conditions, also had higher amounts of total chlorophyll. While C. dactylon "Satiri," C. dactylon "Tifdwarf," and D. didactyla had higher total chlorophyll under normal conditions, $P$. vaginatum, and Z. japonica maintained comparatively higher amounts of total chlorophyll under salt stress with marginal reductions compared to other turf species.

3.7. Root Cell Histology. Differences in cell damage to root cortex of turfgrass species were observed. The damage resulted from cell collapse due to salt stress. Cortical cell of $P$. vaginatum, and $Z$. japonica did not show cell collapse in 24 and $48 \mathrm{dS} \mathrm{m}^{-1}$ salinity treatments (Figures 3 and 4). Zoysia matrella showed less cell collapse at $48 \mathrm{dS} \mathrm{m}^{-1}$ salinity treatment (Figure 5). Digitaria didactyla, C. dactylon "Tifdwarf," and C. dactylon "Satiri" showed severe cell collapse at the highest salinity level $\left(48 \mathrm{dS} \mathrm{m}^{-1}\right)$ compared to the control (Figures 6, 7, and 8).

\section{Discussion}

The six turfgrass species in the present study exhibited a wide range in salinity tolerance in terms of dry matter production (Figures 1 and 2) and organic osmolyte accumulation (Table 4). In Malaysia, such type of research was not conducted ever before. Previously, we identified turfgrass species that were available in Malaysia and studied growth 


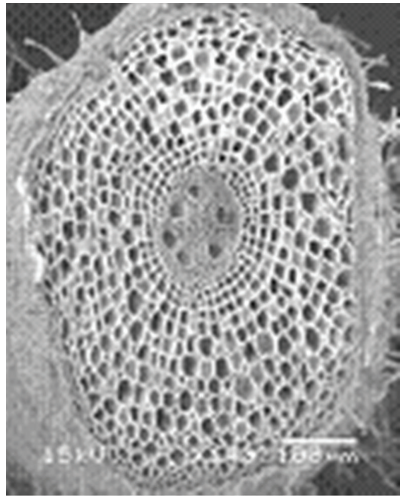

(a)

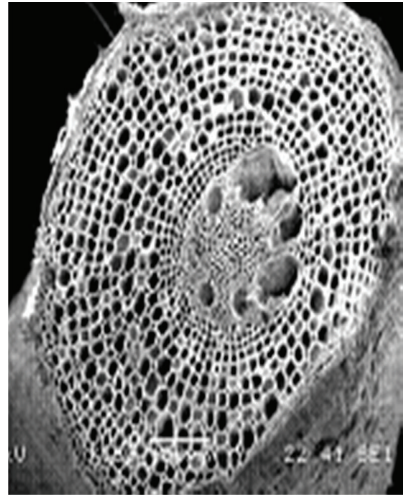

(b)

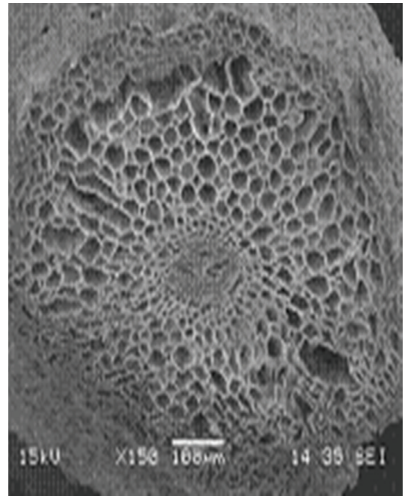

(c)

Figure 3: Scanning electron microscopy photographs showing root cortical tissue of Paspalum vaginatum under (a) 0, (b) 24, and (c) $48 \mathrm{dS} \mathrm{m}^{-1}$.

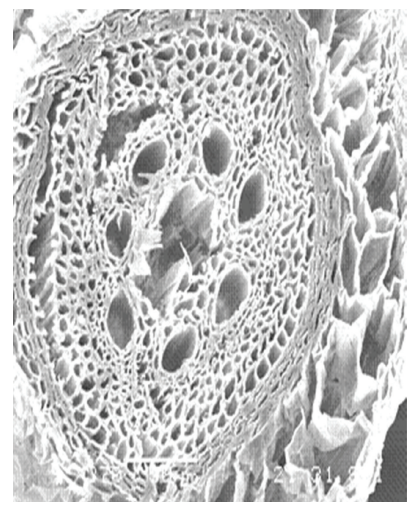

(a)

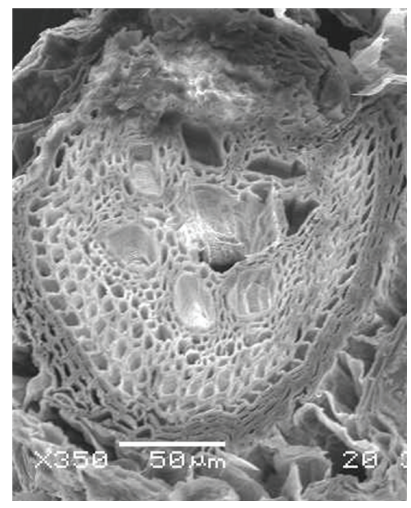

(b)

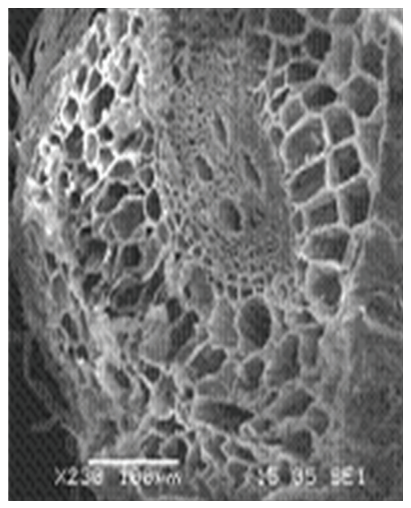

(c)

FIGURE 4: Scanning electron microscopy photographs showing root cortical tissue of Zoysia japonica under (a) 0, (b) 24, and (c) $48 \mathrm{dS} \mathrm{m}^{-1}$.

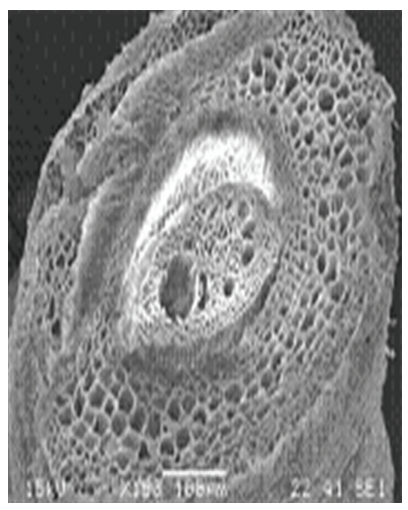

(a)

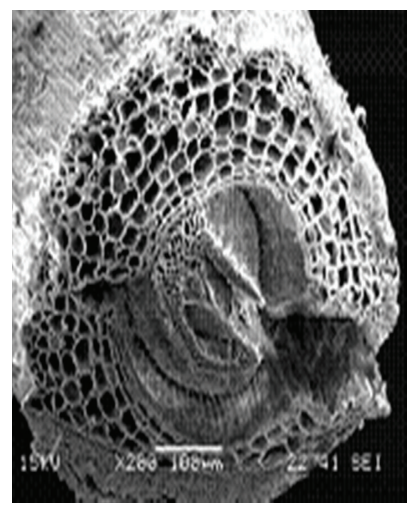

(b)

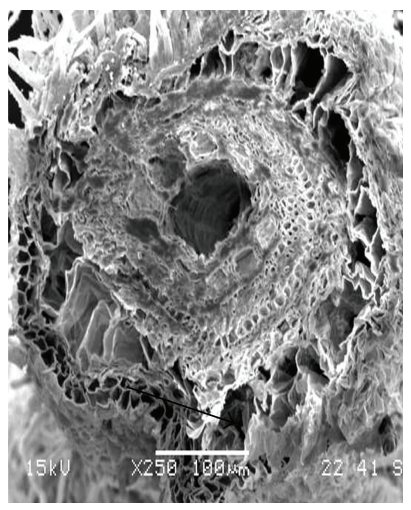

(c)

FIGURE 5: Scanning electron microscopy photographs showing root cortical tissue of Zoysia matrella under (a) 0, (b) 24, and (c) $48 \mathrm{dS} \mathrm{m}^{-1}$.

performance under salinity-stressed conditions [13, 14]. Throughout the globe, seashore paspalum exhibits a wide range of salinity tolerance among ecotypes [19-22]. A wide intraspecific variation in salinity tolerance has been reported to be as great as the interspecific variations [23]. Several researchers have reported that halophytes, which are ion includers, often adapt to low water potential by accumulation of inorganic solutes to maintain turgor pressure and total water potential [24-26].

Salinity stressed plants certainly face osmotic challenges. This is in agreement with several previous reports $[5,19,20,27]$, which concur that osmotic adjustment is 


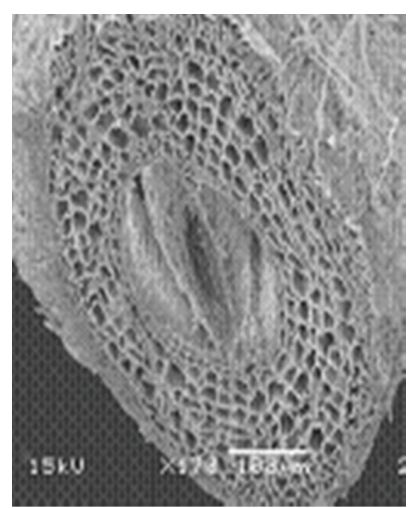

(a)

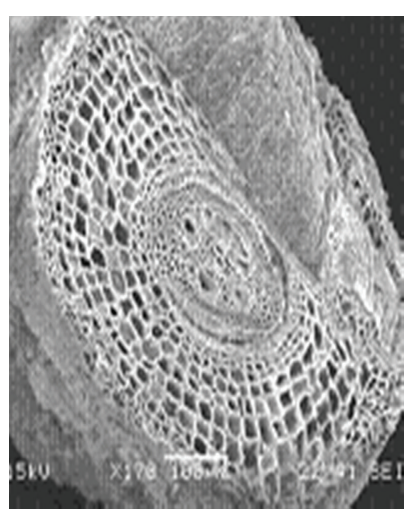

(b)

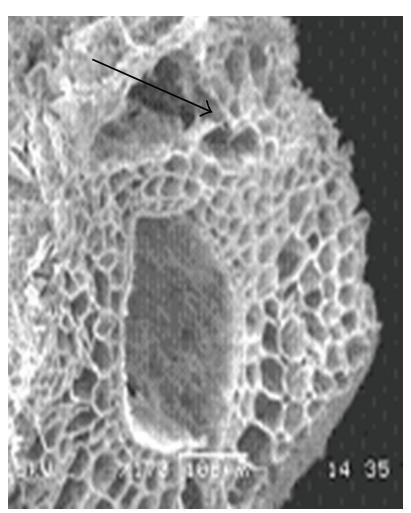

(c)

FIgURE 6: Scanning electron microscopy photographs showing root cortical tissue of C. dactylon "Tifdwarf” under (a) 0, (b) 24, and (c) $48 \mathrm{dS} \mathrm{m}^{-1}$. The arrow indicates cell damage (c) compared to control (a).

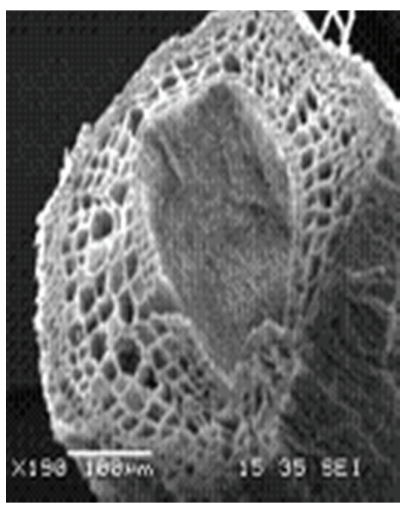

(a)

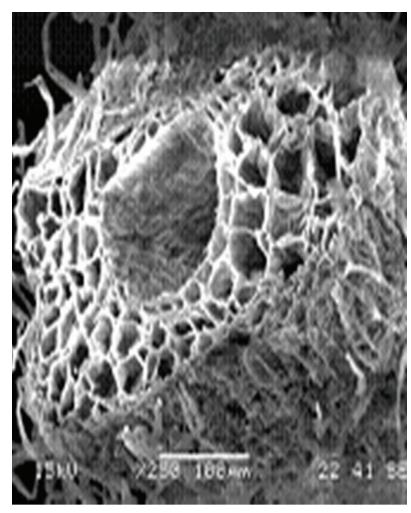

(b)

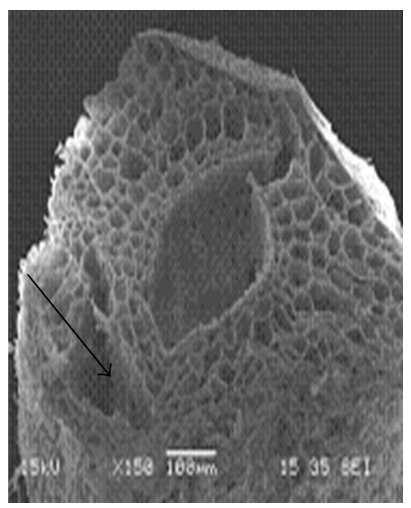

(c)

FIgURE 7: Scanning electron microscopy photographs showing root cortical tissue of C. dactylon "Satiri" under (a) 0, (b) 24, and (c) $48 \mathrm{dS} \mathrm{m}^{-1}$. The arrow indicates cell damage (c) compared to control (a).

the main mechanism for survival and growth of plants under salinity stress. The percentage relative water content (RWC) was determined as an indicator of osmotic status of turfgrass species studied (Table 5). Halophytes are often able to accumulate high charges of salts in their tissues for osmotic adjustment through the compartmentalization of ions in vacuoles and the production of compatible solutes, or osmotic, in the cytoplasm [27]. Some compatible solutes that show an increase in concentration under salinity stress may also play significant role in osmotic adjustment, and these include proline, glycine betaine, and sugars [28-31]. Glycine betaine and proline protect enzymes (proteins) from damages caused by salinity or dehydration stress [32, 33]. Interestingly, significant proline accumulation generally occurs only after exceeding a threshold of drought or salt stress [30]. In the current study, salinity triggered proline synthesis in response to salinity to turgor maintenance (Table 4). Osmotic adjustment through synthesis of organic compounds has been postulated to have a significant role in salt tolerance in P. vaginatum [34]. Our studies indicated that salinity damaged root structure as a result of cortical cell collapse in C. dactylon "Tifdwarf," D. didactyla, and C. dactylon "Satiri." The structural damage in cortical tissue would interrupt radial water movement in the roots, thus limiting water uptake [35].

Chlorophyll degradation is the primary cause of photosynthetic degeneration/leaf firing and a main biochemical factor for the observed growth reduction [36]. The NaClinduced decrease in chlorophyll level is widely reported in both glycophytes and halophytes [37-39]. In the present study, the chlorophyll damage was not recorded until $24 \mathrm{dS} \mathrm{m}^{-1}$ salinity level and thereafter chlorophyll damage increased with increasing salinity (Tables 6, 7, and 8). The chlorophyll degradation is associated with leaf firing (Table 3). Salinity-induced chlorophyll reduction may be related either to $\mathrm{Mg}$ deficiency and/or chlorophyll oxidation since reactive oxygen species (ROS) generation is common in salinity stressed conditions [40]. The chlorophyll-a content of all species decreased much more with increasing salinity (Table 6). However, [41] observed that salinity decreased chlorophyll-b content much more than chlorophyll-a. Chlorophyll content of $P$. vaginatum and $Z$. japonica seem to be insensitive to salinity up to $48 \mathrm{dS} \mathrm{m}^{-1}$. This is consistent with the earlier reports for other monocots including rice, 


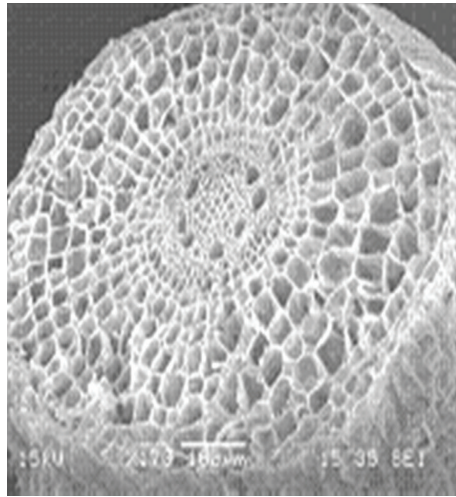

(a)

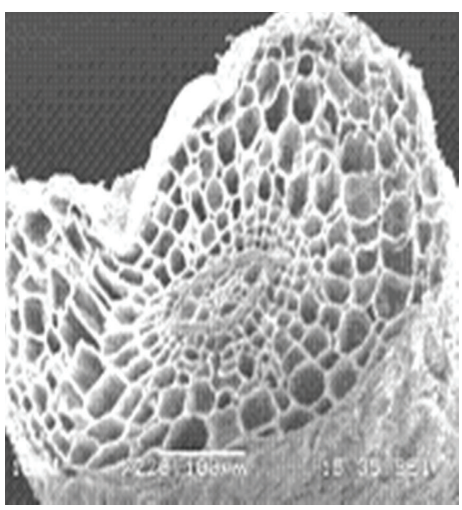

(b)

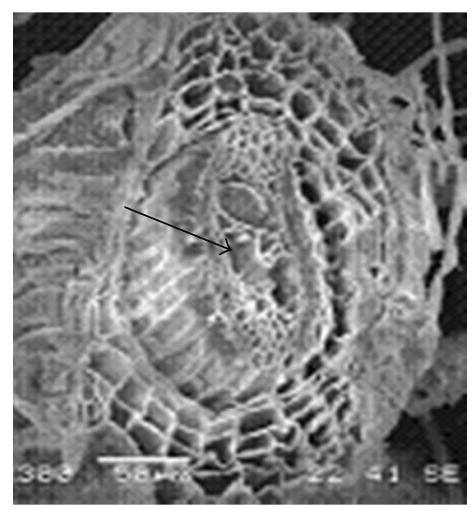

(c)

Figure 8: Scanning electron microscopy photographs showing root cortical tissue of Digitaria didactyla under (a) 0, (b) 24, and (c) $48 \mathrm{dS} \mathrm{m}^{-1}$. The arrow indicates cell damage (c) compared to control (a).

wheat and maize chlorophyll-a by [42-44], chlorophyll-b and total chlorophyll contents decreased with increasing salinity [45], and salt-sensitive rice cultivars had lower chlorophyll content than salt-tolerant rice cultivars [45]. Similar observations were made by $[46,47]$.

\section{Conclusion}

The development of turfgrass industry in the coastal areas of Malaysia is challenging due to scarcity of fresh water for irrigation and salt tolerant weed species infestation. Sea water irrigation is a new technology widely used to suppress weed and maintaining the turfgrass growth simultaneously. Appropriate, realistic physiological criteria are essential to define the salinity tolerance and growth responses of turfgrass species. In the present study, salinity tolerance was evaluated on the basis of leaf firing, shoot and root growth reduction, proline content, and relative water content. We observed that $P$. vaginatum was highly salt tolerant at $48 \mathrm{dS} \mathrm{m}^{-1}$ followed by Z. japonica and Z. matrella, while $C$. dactylon "Tifdwarf" was least salt tolerant followed by $D$. didactyla and C. dactylon "Satiri." The conclusions are based on responses of six turfgrass species to salinity. Many of the principles can be employed to discuss issues related to development of better direct selection criteria for other turfgrass species.

\section{Acknowledgments}

Authors would like to acknowledge funding of this work by Malaysian Government Research Grant (Science Fund 0501-04 SF0302) and University Putra Malaysia for Graduate Research Fellowship.

\section{References}

[1] L. B. McCarty and A. E. Dudeck, "Salinity effects on bentgrass germination," HortScience, vol. 28, no. 1, pp. 15-17, 1993.

[2] C. L. Murdoch, "Water the limiting factor for golf course development in Hawaii," U.S.G.A. Green Section Record, vol. 25, pp. 11-13, 1987.
[3] A. Harivandi, J. D. Bulter, and L. Wu, "Salinity and turfgrass culture," in Turfgrass, D. V. Waddington, R. N. Carrow, and R. C. Shearman, Eds., Agronomy Monograph, pp. 208-230, ASA. CSSA and SSSA, Madison, Wis, USA, 1992.

[4] A. C. Hixson, W. T. Crow, R. McSorley, and L. E. Trenholm, "Saline irrigation affects Belonolaimus longicaudatus and Hoplolaimus galeatus on seashore paspalum," Journal of Nematology, vol. 37, no. 1, pp. 37-44, 2005.

[5] R. Munns and M. Tester, "Mechanisms of salinity tolerance," Annual Review of Plant Biology, vol. 59, pp. 651-681, 2008.

[6] K. B. Marcum, "Use of saline and non-potable water in the turfgrass industry: constraints and developments," Agricultural Water Management, vol. 80, no. 1-3, pp. 132-146, 2006.

[7] D. Rhodes, A. Nadolska-Orczyk et al., "Salinity, osmolytes and compatible solutes," in Salinity: Environment-PlantsMolecules, A. Lauchli and U. Luttge, Eds., Kluwer Academic, Boston, Mass, USA, 2002.

[8] O. Borsani, V. Valpuesta, and M. A. Botella, "Developing salt tolerant plants in a new century: a molecular biology approach," Plant Cell, Tissue and Organ Culture, vol. 73, no. 2, pp. 101-115, 2003.

[9] R. K. Sairam, A. Tyagi, and V. Chinnusamy, "Salinity tolerance: cellular mechanisms and gene regulation," in PlantEnvironment Interactions, B. Huang, Ed., CRC Press, Boca Raton, Fla, USA, 3rd edition, 2006.

[10] B. Jacoby, "Mechanism involved in salt tolerance of plants," in Handbook of Plant and Crop Stress, M. Pessarakli, Ed., pp. 97124, Marcel Dekker, Inc., New York, NY, USA, 1999.

[11] M. K. Kamal-Uddin, A. S. Juraimi, M. Begum, M. R. Ismail, A. A. Rahim, and R. Othman, "Floristic composition of weed community in turf grass area of West Peninsular Malaysia," International Journal of Agriculture and Biology, vol. 11, no. 1, pp. 13-20, 2009.

[12] M. K. Uddin, A. S. Juraimi, M. R. Ismail, and J. T. Brosnan, "Characterizing weed populations in different turfgrass sites throughout the Klang Valley of Western Peninsular Malaysia," Weed Technology, vol. 24, no. 2, pp. 173-181, 2010.

[13] M. K. Uddin, A. S. Juraimi, M. R. Ismail, R. Othman, and A. A. Rahim, "Growth response of eight tropical turfgrass species to salinity," African Journal of Biotechnology, vol. 8, no. 21, pp. 5799-5806, 2009.

[14] M. K. Uddin, A. S. Juraimi, M. R. Ismail, R. Othman, and A. A. Rahim, "Relative salinity tolerance of warm season turfgrass 
species," Journal of Environmental Biology, vol. 32, no. 3, pp. 309-312, 2011.

[15] S. F. Alshammary, Y. L. Qian, and S. J. Wallner, "Growth response of four turfgrass species to salinity," Agricultural Water Management, vol. 66, no. 2, pp. 97-111, 2004.

[16] L. S. Bates, R. P. Waldren, and I. D. Teare, "Rapid determination of free proline for water-stress studies," Plant and Soil, vol. 39, no. 1, pp. 205-207, 1973.

[17] F. H. Witham, D. F. Blaydes, and R. M. Devlin, Exercises in Plant Physiology, PWS, Boston, Mass, USA, 2nd edition, 1986.

[18] P. E. Whetherly, "Studies in the water relation of cotton plants. The field measurement of water deficit in leaves," New Phytology, vol. 49, pp. 81-87, 1950.

[19] G. Lee, R. N. Carrow, and R. R. Duncan, "Photosynthetic responses to salinity stress of halophytic seashore paspalum ecotypes," Plant Science, vol. 166, no. 6, pp. 1417-1425, 2004.

[20] G. Lee, R. N. Carrow, and R. R. Duncan, "Salinity tolerance of seashore paspalum ecotypes: shoot growth responses and criteria," HortScience, vol. 39, no. 5, pp. 1138-1142, 2004.

[21] G. Lee, R. N. Carrow, and R. R. Duncan, "Criteria for assessing salinity tolerance of the halophytic turfgrass seashore paspalum," Crop Science, vol. 45, no. 1, pp. 251-258, 2005.

[22] G. Lee, R. N. Carrow, and R. R. Duncan, "Growth and water relation responses to salinity stress in halophytic seashore paspalum ecotypes," Scientia Horticulturae, vol. 104, no. 2, pp. 221-236, 2005.

[23] M. W. Hester, I. A. Mendelssohn, and K. L. McKee, "Species and population variation to salinity stress in Panicum hemitomon, Spartina patens, and Spartina alterniflora: morphological and physiological constraints," Environmental and Experimental Botany, vol. 46, no. 3, pp. 277-297, 2001.

[24] E. P. Glenn, "Relationship between cations accumulation and water content of salt-tolerant grasses and a sedg," Plant Cell Environment, vol. 10, pp. 205-212, 1987.

[25] T. J. Flowers, S. A. Flowers, M. A. Hajibagheri, and A. R. Yeo, "Salt tolerance in the halophytic wild rice, Porteresia coantata," New Phytology, vol. 114, pp. 675-684, 1990.

[26] E. P. Glenn, M. C. Watson, J. W. O’Leary, and R. D. Axelson, "Comparison of salt tolerance and osmotic adjustment of lowsodium and high-sodium subspecies of the $\mathrm{C}_{4}$ halophytes, Atriplex canescens," Plant Cell Environment, vol. 15, no. 6, pp. 711-718, 1992.

[27] J. Gorham, R. G. W. Jones, and E. McDonnell, "Some mechanisms of salt tolerance in crop plants," Plant and Soil, vol. 89, no. 1-3, pp. 15-40, 1985.

[28] R. G. Storey and R. W. Jones, "Response of Atriplex spongiosa and Suaeda monoica to salinity," Plant Physiology, vol. 63, no. 1, pp. 156-162, 1979.

[29] M. Briens and F. Larher, "Osmoregulation in halophytic higher plants: a comparative study of soluble carbohydrates, polyols, betaines and free proline," Plant Cell Environment, vol. 5, no. 4, pp. 287-292, 1982.

[30] A. J. Cavalieri and A. H. C. Huang, "Evaluation of proline accumulation in the adaptation of diverse species of marsh halophytes to the saline environment," American Journal of Botany, vol. 66, no. 3, pp. 307-312, 1979.

[31] A. J. Cavalieri and A. H. C. Huang, "Accumulation of proline and glycinebetaine in Spartina alterniflora Loisel. in response to $\mathrm{NaCl}$ and nitrogen in the marsh," Oecologia, vol. 49 , no. 2, pp. 224-228, 1981.

[32] L. G. Paleg, G. R. Stewart, and J. W. Bradbeer, "Proline and glycine betaine influence protein salvation," Plant Physiology, vol. 75, no. 4, pp. 974-978, 1984.
[33] N. Smirnoff and Q. J. Cumbes, "Hydroxyl radical scavenging activity of compatible solutes," Phytochemistry, vol. 28, no. 4, pp. 1057-1060, 1989.

[34] K. B. Marcum and C. L. Murdoch, "Salinity tolerance mechanisms of six $\mathrm{C}_{4}$ turfgrasses," Journal of the American Society for Horticultural Science, vol. 119, no. 4, pp. 779-784, 1994.

[35] M. G. Huck, B. L. Klepper, and H. M. Taylor, "Diurnal variations in root diameter," Plant Physiology, vol. 45, no. 4, pp. 529-530, 1970.

[36] J. D. Everard, R. Gucci, S. C. Kann, J. A. Flore, and W. H. Loescher, "Gas exchange and carbon partitioning in the leaves of celery (Apium graveolens L.) at various levels of root zone salinity," Plant Physiology, vol. 106, no. 1, pp. 281-292, 1994.

[37] Z. Abdullah, M. A. Khan, and T. J. Flowers, "Causes of sterility in seed set of rice under salinity stress," Journal of Agronomy and Crop Science, vol. 187, no. 1, pp. 25-32, 2001.

[38] C. Kaya, A. L. Tuna, M. Ashraf, and H. Altunlu, "Improved salt tolerance of melon (Cucumis melo L.) by the addition of proline and potassium nitrate," Environmental and Experimental Botany, vol. 60, no. 3, pp. 397-403, 2007.

[39] L. Shabala, T. A. Cuin, I. A. Newman, and S. Shabala, "Salinityinduced ion flux patterns from the excised roots of Arabidopsis sos mutants," Planta, vol. 222, no. 6, pp. 1041-1050, 2005.

[40] F. Moradi and A. M. Ismail, "Responses of photosynthesis, chlorophyll fluorescence and ROS-scavenging systems to salt stress during seedling and reproductive stages in rice," Annals of Botany, vol. 99, no. 6, pp. 1161-1173, 2007.

[41] M. S. Islam, Morpho-physiology of blackgram and mungbean as influenced by salinity, M.S. thesis, Department of Agronomy, BSMRAU, Salna, Gazipur, 2001.

[42] S. Lutts, J. M. Kinet, and J. Bouharmont, "NaCl-induced senescence in leaves of rice (Oryza sativa L.) cultivars differing in salinity resistance," Annals of Botany, vol. 78, no. 3, pp. 389398, 1996.

[43] S. Krishna, B. T. Raj, E. C. Mawson, T. Yeung, and A. Thorpe, "Utilization of induction and quenching kinetics of chlorophyll a fluorescence for in vivo salinity screening studies in wheat (Triticum aestivum vars. Kharchia-65 and Fielder)," Canadian Journal of Botany, vol. 71, no. 1, pp. 87-92, 1993.

[44] S. N. Shabala, S. I. Shabala, A. I. Martynenko, O. Babourina, and I. A. Newman, "Salinity effect on bioelectric activity, growth, $\mathrm{Na}^{+}$accumulation and chlorophyll fluorescence of maize leaves: a comparative survey and prospects for screening," Australian Journal of Plant Physiology, vol. 25, no. 5, pp. 609-616, 1998.

[45] R. Pushpam and S. R. S. Rangasamy, "In vivo response of rice cultivars to salt stress," Journal of Ecobiology, vol. 14, pp. 177182, 2002.

[46] M. M. Mohan, S. L. Narayanan, and S. M. Ibrahim, "Chlorophyll stability index (CSI): its impact on salt tolerance in rice," International Rice Research Notes, vol. 25, no. 2, pp. 38-39, 2000.

[47] M. P. Mandal and R. A. Singh, "Impact of salt stress on chlorophyll content in rice genotypes," Journal Research Birsa Agriculture University India, vol. 13, pp. 61-63, 2001. 

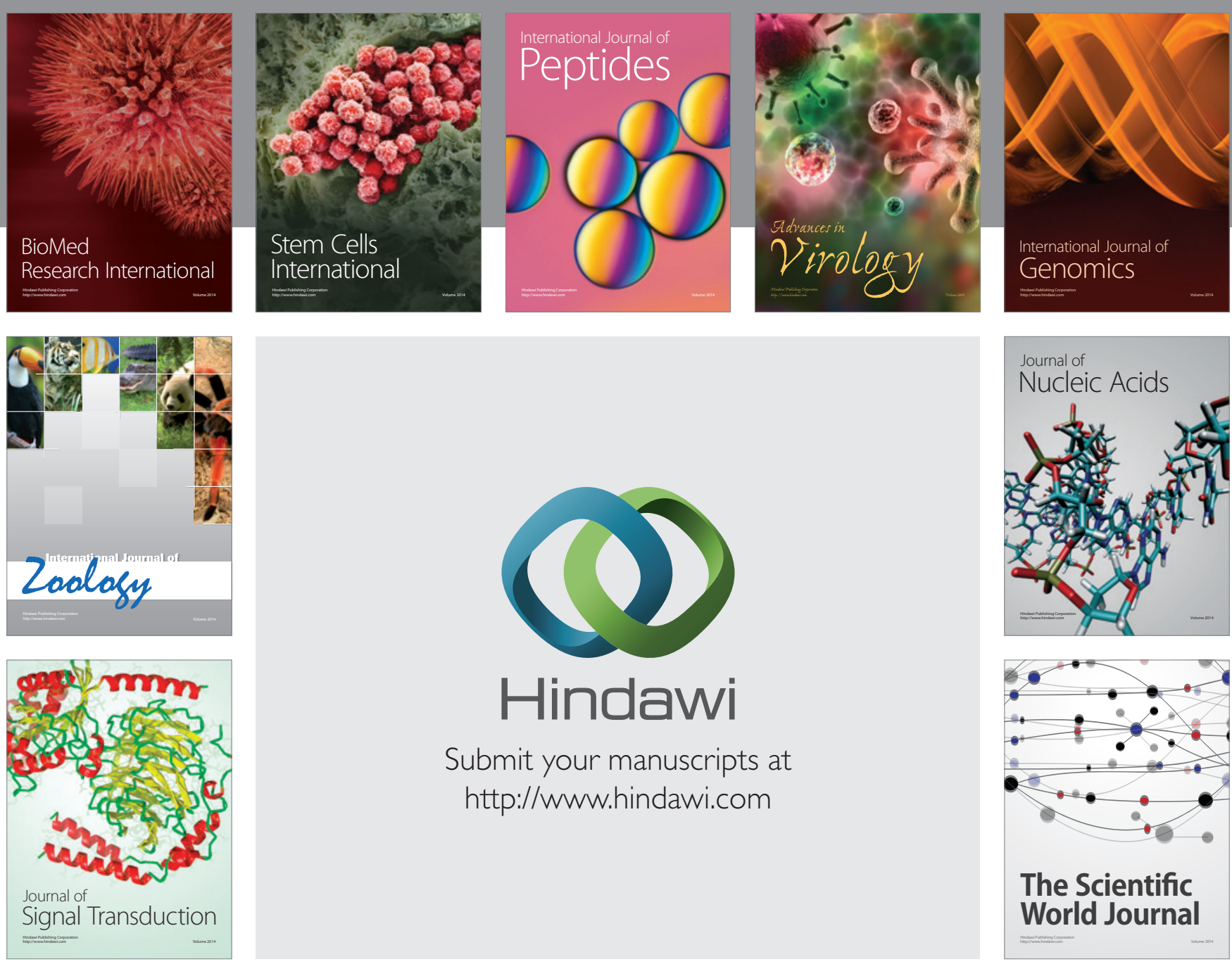

Submit your manuscripts at

http://www.hindawi.com
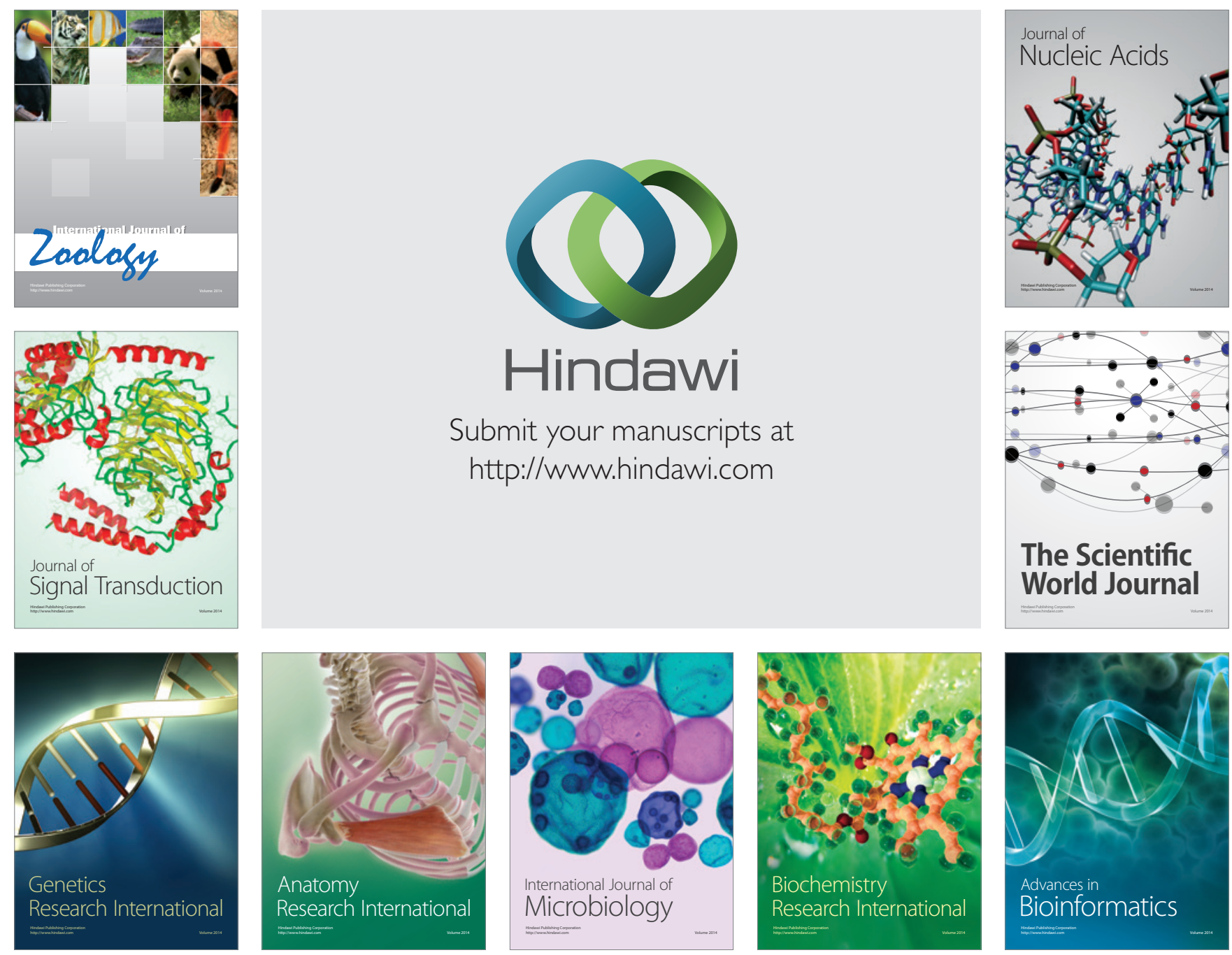

The Scientific World Journal
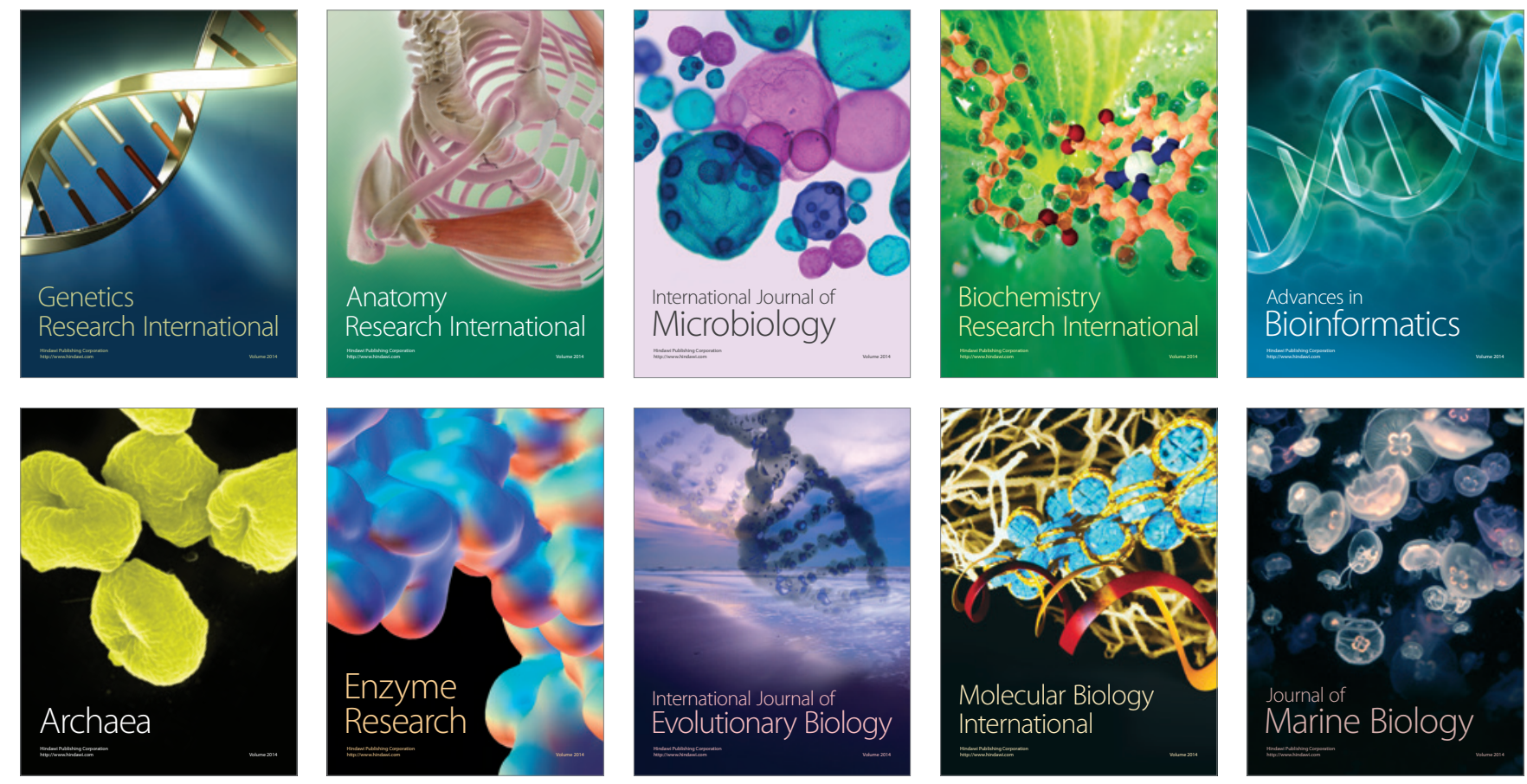